\title{
Asteroseismology from Dome A, Antarctica
}

\author{
J. N. Fu ${ }^{1}$, W. K. Zong ${ }^{1}$, Y. Yang ${ }^{2,1}$, A. Moore ${ }^{3}$, M. C. B. Ashley ${ }^{4}$, \\ X. Q. Cui ${ }^{5}$, L. L. Feng ${ }^{6}$, X. F. Gong ${ }^{5}$, J. S. Lawrence ${ }^{4,7}$, \\ D. Luong-Van ${ }^{4}$, Q. Liu ${ }^{8}$, C. R. Pennypacker ${ }^{9}$, Z. H. Shang ${ }^{10}$, \\ J. W. V. Storey ${ }^{4}$, L. Z. Wang ${ }^{8}$, L. F. Wang ${ }^{6,2}$, H. G. Yang ${ }^{11}$,

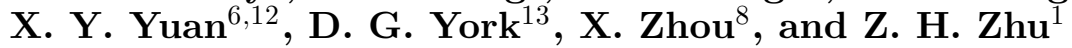 \\ ${ }^{1}$ Department of Astronomy, Beijing Normal University, Beijing 100875, China \\ ${ }^{2}$ Department of Physics and Astronomy, Texas A\&M University, College Station 77843, USA \\ ${ }^{3}$ Caltech Optical Observatories, California Institute of Technology, Pasadena CA 91107, USA \\ ${ }^{4}$ School of Physics, University of New South Wales, NSW 2052, Australia \\ ${ }^{5}$ Nanjing Institute of Astronomical Optics and Technology, Nanjing 210042, China \\ ${ }^{6}$ Purple Mountain Observatory, Chinese Academy of Sciences, Nanjing 210008, China \\ ${ }^{7}$ Australian Astronomical Observatory, NSW 1710, Australia \\ ${ }^{8}$ National Astronomical Obs. of China, Chinese Academy of Sciences, Beijing 100012, China \\ ${ }^{9}$ Center for Astrophysics, Lawrence Berkeley National Laboratory, Berkeley, CA, USA \\ ${ }^{10}$ Tianjin Normal University, Tianjin 300074, China \\ ${ }^{11}$ Polar Research Institute of China, Pudong, Shanghai 200136, China \\ ${ }^{12}$ Chinese Center for Antarctic Astronomy, Nanjing 210008, China \\ ${ }^{13}$ Department of Astronomy and Astrophysics and Enrico Fermi Institute, University of \\ Chicago, Chicago, IL 60637, USA
}

\begin{abstract}
Gattini and CSTAR have been installed at Dome A, Antarctica, which provide timeseries photometric data for a large number of pulsating variable stars. We present the study for several variable stars with the data collected with the two facilities in 2009 to demonstrate the scientific potential of observations from Dome A for asteroseismology.
\end{abstract}

\section{Introduction}

Observations in polar sites open a new window for long, uninterrupted and consecutive time-series photometry/spectroscopy for pulsating variable stars, a powerful tool for asteroseismology. Dome A, located at longitude $77^{\circ} 06^{\prime} 57^{\prime \prime} \mathrm{E}$ and latitude $80^{\circ} 25^{\prime} 08^{\prime \prime} \mathrm{S}$ with a 4013-m elevation as the highest point on the Antarctic plateau, is widely predicted to be a very good astronomical site (Saunders et al. 2009). The first-generation facilities including Gattini (Moore et al. 2013) and CSTAR (Chinese Small Telescope ARray, Liu \& Yuan 2009) were deployed on Dome A in 2008 January. We present some progress on asteroseismology, using the time-series multi-color photometric data collected with the two telescopes in the winter of 2009.

\section{Bright variable stars observed with Gattini}

Gattini is a $10.5-\mathrm{mm}$ fish-eye lens equipped with an Apogee $2 \mathrm{k} \times 2 \mathrm{k}$ CCD camera. A filter rotator allows frames taken in Johnson $B V R$ and a band of $\mathrm{OH}$ in turn. The field of view is $90^{\circ}$. In 2009 winter, a total of $377 \mathrm{~GB}$ of data were taken. After data reduction and stellar photometry, light curves of 66 variable stars were obtained. Figure 1 shows the light curves of a $\mathrm{W}$ Vir type variable star $\kappa \mathrm{Pav}(V=4.4 \mathrm{mag})$ in $B, V, R$ in 2009. 


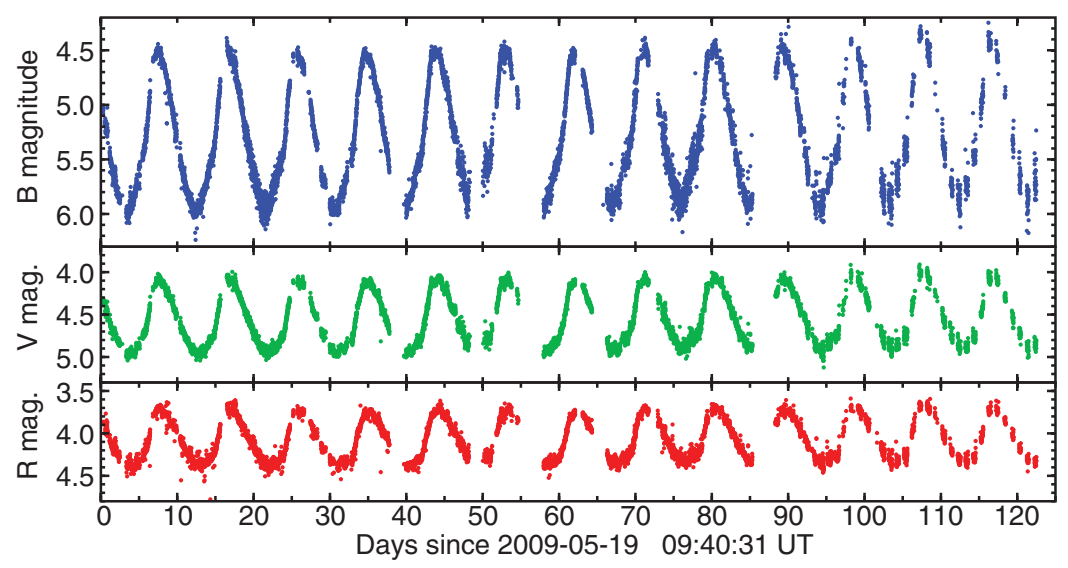

Figure 1. Light curves of $\kappa$ Pav in $B, V$ and $R$, data from 2009.

\section{Variable stars observed with CSTAR}

CSTAR has four telescopes with apertures of $15-\mathrm{cm}$, equipped with four $1 \mathrm{k} \times 1 \mathrm{k}$ CCD cameras. The filters of SDSS $g^{\prime}, r^{\prime}$ and $i^{\prime}$ are mounted to three of them while the fourth telescope has no filter. The pointing was fixed to the celestial southern pole with field of view of $4.5^{\circ} \times 4.5^{\circ}$. Times-series CCD frames were taken in $g^{\prime}$ and $r^{\prime}$ in 2009 winter.

The light curves of HD $92277(V=9.10 \mathrm{mag}, B-V=0.38 \mathrm{mag})$ show that it is a new variable star. A total of 13 and 24 independent frequencies are detected in $g^{\prime}$ and $r^{\prime}$, respectively. Combining with the available parameters of the star, we classify it as a new $\delta$ Scuti star (Zong et al. 2013). In addition, light curves of three RR Lyrae stars in the field of view of CSTAR were obtained in $g^{\prime}$ and $r^{\prime}$ in 2009.

\section{Summary}

Variable stars have been studied with the time-series photometric data collected from Dome A, Antarctica. In an epoch of space missions (e.g. CoRoT and Kepler) which are providing high duty-cycle high precision time-series photometry for variables, observations for pulsating stars can be made through multiple colors simultaneously from Dome A, Antarctica, which provides important information for pulsation mode identification. This might bring a chance of a new break-through in asteroseismology.

\section{Acknowledgement}

This work is supported by the National Basic Research Program of China (973 Program 2013CB834900).

\section{References}

Liu, G. \& Yuan, X. 2009, Acta Astron. Sinica, 50, 224

Moore, A., Yang, Y., Fu, J. N., et al. 2013, in: M. G. Burton, X. Cui, \& N. F. H. Tothill (eds.), Astrophysics from Antarctica, Proc. IAU Symposium No. 288 (Cambridge: Cambridge University Press), p. 34

Saunders, W., Lawrence, J. S., Storey, J. W. V., et al. 2009, PASP, 121, 976

Zong, W. K., Fu, J. N., Niu, J. S., et al. 2013, AJ, submitted 